\title{
WKB Approximation in Noncommutative Gravity
}

Maja BURIĆ ${ }^{\dagger}$, John MADORE ${ }^{\ddagger}$ and George ZOUPANOS ${ }^{\S}$

$\dagger$ Faculty of Physics, University of Belgrade, P.O. Box 368 RS-11001 Belgrade, Serbia

E-mail: majab@phy.bg.ac.yu

¥ Laboratoire de Physique Théorique, Université de Paris-Sud, Bâtiment 211, F-91405 Orsay, France

E-mail: madore@th.u-psud.fr

$\S$ Physics Department, National Technical University, Zografou Campus, GR-15780 Athens

E-mail:zoupanos@cern.ch

Received October 25, 2007, in final form December 21, 2007; Published online December 24, 2007

Original article is available at http://www.emis.de/journals/SIGMA/2007/125/

\begin{abstract}
We consider the quasi-commutative approximation to a noncommutative geometry defined as a generalization of the moving frame formalism. The relation which exists between noncommutativity and geometry is used to study the properties of the highfrequency waves on the flat background.
\end{abstract}

Key words: noncommutative geometry; models of quantum gravity

2000 Mathematics Subject Classification: 46L87; 83C35

\section{Preliminary formalism}

Our purpose in this paper is to analyze the relation which exists between the noncommutativity of the local coordinates and the gravitational field on a given space-time. In particular model of noncommutative gravity which we develop, in the noncommutative frame formalism, this relation is expressed as consistency between the algebraic and the differential-geometric structures, or in other language as generalized Jacobi identities.

Let $\mu$ be a typical 'large' source mass with 'Schwarzschild radius' $G_{N} \mu$. We have two length scales, determined by respectively $G_{N} \hbar$, the square of the Planck length and by $k$, the scale of noncommutativity. The gravitational field is weak if the dimensionless parameter $\epsilon_{G F}=$ $G_{N} \hbar^{-1} \mu^{2}$ is small; the space-time is almost commutative if the dimensionless parameter $\epsilon=\hbar \mu^{2}$ is small. These two parameters are not necessarily related but we shall here assume that they are of the same order of magnitude,

$\epsilon_{G F} \simeq \epsilon$.

If noncommutativity is not directly related to gravity then it makes sense to speak of ordinary gravity as the limit $k \rightarrow 0$ with $G_{N} \mu$ nonvanishing. On the other hand if noncommutativity and gravity are directly related then both should vanish with $k$. We wish here to consider an expansion in the parameter $\epsilon$, which we have seen is a measure of the relative dimension of a typical 'space-time cell' compared with the Planck length of a typical quantity of gravitational energy. Our motivation for considering noncommutative geometry as an 'avatar' of gravity is the belief that it sheds light on the role of the gravitational field as the universal regulator of ultra-violet divergences. We mention here only some elements of the approach we use to

\footnotetext{
*This paper is a contribution to the Proceedings of the Seventh International Conference "Symmetry in Nonlinear Mathematical Physics" (June 24-30, 2007, Kyiv, Ukraine). The full collection is available at http://www.emis.de/journals/SIGMA/symmetry2007.html
} 
study gravitational fields on Lorentz-signature manifolds. A general description can be found elsewhere [1] as can a simple explicit solution [2]. Some properties of the perturbation analyzed here which we shall use are derived in [3].

We start with a 'noncommutative space', a $*$-algebra $\mathcal{A}$ generated by four Hermitian elements $x^{\mu}$ which satisfy the commutation relations

$$
\left[x^{\mu}, x^{\nu}\right]=i k J^{\mu \nu}\left(x^{\sigma}\right) \text {. }
$$

We assume that algebra is associative, therefore the commutators obey the Jacobi identities. We assume further that over $\mathcal{A}$ there is a differential calculus [1] which possesses a preferred frame $\theta^{\alpha}$, a set of 1 -forms which commute with the algebra,

$$
\left[x^{\mu}, \theta^{\alpha}\right]=0 .
$$

The space one obtains in the commutative limit has a global moving frame $\tilde{\theta}^{\alpha}$. The differential is defined as

$$
d f=e_{\alpha} f \theta^{\alpha}
$$

that is,

$$
d x^{\mu}=e_{\alpha}^{\mu} \theta^{\alpha}, \quad e_{\alpha}^{\mu}=e_{\alpha} x^{\mu} .
$$

The $e_{\alpha}$ are the vector fields dual to the frame forms

$$
\theta^{\alpha}\left(e_{\beta}\right)=\delta_{\beta}^{\alpha}
$$

We have two basic structures: the algebra $\mathcal{A}$ defined by a product which is restricted by the matrix of elements $J^{\mu \nu}$ and the metric defined by a frame that is the matrix of elements $e_{\alpha}^{\mu}$. Consistency requirements impose relations between these two structures which in simple situations allow us to find a one-to-one correspondence between the commutators and the metric. Most important relation is the Leibniz rule

$$
i \hbar d J^{\mu \nu}=\left[d x^{\mu}, x^{\nu}\right]+\left[x^{\mu}, d x^{\nu}\right]=\left[e_{\alpha}^{\mu}, x^{\nu}\right] \theta^{\alpha}-\left[e_{\alpha}^{\nu}, x^{\mu}\right] \theta^{\alpha} .
$$

One can see in (1) a differential equation for $J^{\mu \nu}$ in terms of $e_{\alpha}^{\mu}$. In important special cases this equation reduces to a simple differential equation of one variable.

We must insure in addition that the differential is well defined. A necessary condition is that $d\left[x^{\mu}, \theta^{\alpha}\right]=0$, from which it follows that

$$
d\left[x^{\mu}, \theta^{\alpha}\right]=\left[d x^{\mu}, \theta^{\alpha}\right]+\left[x^{\mu}, d \theta^{\alpha}\right]=e_{\beta}^{\mu}\left[\theta^{\beta}, \theta^{\alpha}\right]-\frac{1}{2}\left[x^{\mu}, C^{\alpha}{ }_{\beta \gamma}\right] \theta^{\beta} \theta^{\gamma} .
$$

We have introduced the Ricci rotation coefficients

$$
d \theta^{\alpha}=-\frac{1}{2} C^{\alpha}{ }_{\beta \gamma} \theta^{\beta} \theta^{\gamma} .
$$

Therefore we find that multiplication of 1-forms satisfies

$$
\left[\theta^{\alpha}, \theta^{\beta}\right]=\frac{1}{2} \theta_{\mu}^{\beta}\left[x^{\mu}, C_{\gamma \delta}^{\alpha}\right] \theta^{\gamma} \theta^{\delta} .
$$

The metric is defined by the map

$$
g\left(\theta^{\alpha} \otimes \theta^{\beta}\right)=g^{\alpha \beta} .
$$


The bilinearity of the metric implies that $g^{\alpha \beta}$ are numbers and not functions of coordinates, exactly as in the commutative case. We choose the frame to be orthonormal; we can write therefore

$$
g^{\alpha \beta}=\eta^{\alpha \beta}
$$

We introduce also

$$
g^{\mu \nu}=g\left(d x^{\mu} \otimes d x^{\nu}\right)=e_{\alpha}^{\mu} e_{\beta}^{\nu} g^{\alpha \beta} .
$$

Other differential-geometric quantities: connection, torsion and curvature can be defined with the same formulae as in commutative differential geometry. We will take here that the torsion vanishes.

\section{The quasi-commutative approximation}

To lowest order in $\epsilon$ the partial derivatives are well defined and the approximation, which we shall refer to as the quasi-commutative,

$$
\left[x^{\lambda}, f\right]=i k J^{\lambda \sigma} \partial_{\sigma} f
$$

is valid. The Leibniz rule and the Jacobi identity can be written in this approximation as

$$
e_{\alpha} J^{\mu \nu}=\partial_{\sigma} e_{\alpha}^{[\mu} J^{\sigma \nu]}, \quad \epsilon_{\kappa \lambda \mu \nu} J^{\gamma \lambda} e_{\gamma} J^{\mu \nu}=0 .
$$

We call these equations the Jacobi equations.

Written in frame components $J^{\alpha \beta}$ of the commutators, $J^{\alpha \beta}=\theta_{\mu}^{\alpha} \theta_{\nu}^{\beta} J^{\mu \nu}$, the Jacobi equations become

$$
\begin{aligned}
& e_{\gamma} J^{\alpha \beta}-C_{\gamma \delta}^{[\alpha} J^{\beta] \delta}=0, \\
& \epsilon_{\alpha \beta \gamma \delta} J^{\gamma \eta}\left(e_{\eta} J^{\alpha \beta}+C^{\alpha}{ }_{\eta \zeta} J^{\beta \zeta}\right)=0 .
\end{aligned}
$$

We have used here the expression for the rotation coefficients, valid also in the quasi-commutative approximation

$$
C_{\beta \gamma}^{\alpha}=\theta_{\mu}^{\alpha} e_{[\beta} e_{\gamma]}^{\mu}=-e_{\beta}^{\nu} e_{\gamma}^{\mu} \partial_{[\nu} \theta_{\mu]}^{\alpha},
$$

and the inverse $\theta_{\mu}^{\alpha}$ of the $e_{\alpha}^{\mu}$.

Equation (2) for the rotation coefficients can be solved. Provided $J_{\alpha \beta}^{-1}$ exists, after some algebra we obtain that

$$
C_{\beta \gamma}^{\alpha}=J^{\alpha \eta} e_{\eta} J_{\beta \gamma}^{-1}
$$

If we introduce

$$
\hat{C}_{\alpha \beta \gamma}=J_{\alpha \delta}^{-1} C_{\beta \gamma}^{\delta},
$$

we find that

$$
\hat{C}_{\alpha \beta \gamma}=e_{\alpha} J_{\beta \gamma}^{-1}
$$

and also

$$
\hat{C}_{\alpha \beta \gamma}+\hat{C}_{\beta \gamma \alpha}+\hat{C}_{\gamma \alpha \beta}=0 .
$$

This equation we can write as a de Rham cocycle condition

$$
d J^{-1}=0, \quad J^{-1}=\frac{1}{2} J_{\alpha \beta}^{-1} \theta^{\alpha} \theta^{\beta} .
$$




\section{The weak-field approximation}

We assumed in the previous section that the noncommutativity is small and we derived some relations to first-order in the parameter $\epsilon$. We shall now make an analogous assumption concerning the gravitational field; we shall assume that $\epsilon_{G F}$ is also small and of the same order of magnitude. With these two assumptions the Jacobi equations become relatively easy to solve.

We suppose that the basic unknowns, the commutators and the frame components are constants in the ground state. That is, the ground state is a flat noncommutative space characterized by $J_{0}^{\mu \nu}$ and $\theta_{0}^{\alpha}$. It is perturbed to

$$
J^{\alpha \beta}=J_{0}^{\alpha \beta}+\epsilon I^{\alpha \beta}, \quad \theta^{\alpha}=\theta_{0}^{\beta}\left(\delta_{\beta}^{\alpha}-\epsilon \Lambda_{\beta}^{\alpha}\right) .
$$

The leading order of the Jacobi system is then given by

$$
\begin{aligned}
& e_{\gamma} I^{\alpha \beta}-e_{[\gamma} \Lambda_{\delta]}^{[\alpha} J_{0}^{\beta] \delta}=0, \\
& \epsilon_{\alpha \beta \gamma \delta} J_{0}^{\gamma \eta} e_{\eta} I^{\alpha \beta}=0 .
\end{aligned}
$$

Introducing the notation

$$
\hat{I}_{\alpha \beta}=J_{0}^{-1}{ }_{\alpha \gamma} J_{0}^{-1}{ }_{\beta \delta}^{\gamma \delta}, \quad \hat{\Lambda}_{\alpha \beta}=J_{0}^{-1}{ }_{\alpha \gamma} \Lambda_{\beta}^{\gamma},
$$

(3) can be written as

$$
e_{\gamma}\left(\hat{I}_{\alpha \beta}-\hat{\Lambda}_{[\alpha \beta]}\right)=e_{[\alpha} \hat{\Lambda}_{\beta] \gamma}
$$

and (4) as

$$
\epsilon^{\alpha \beta \gamma \delta} e_{\alpha} \hat{I}_{\beta \gamma}=0 .
$$

We note that $\hat{I}$ is a linear perturbation of $J_{0}^{-1}$,

$$
J_{\alpha \beta}^{-1}=J_{0 \alpha \beta}^{-1}+\epsilon \hat{I}_{\alpha \beta} .
$$

Equations (5)-(6) can be solved [3]. The most general solution is given by

$$
\hat{\Lambda}_{\alpha \beta}=\hat{I}_{\alpha \beta}+e_{\beta} A_{\alpha}+c_{\alpha \beta},
$$

where $c$ is a 2 -form with constant components and $A$ is an arbitrary 1-form. $\hat{I}$ must obey the cocycle condition (4) which, introducing

$$
\hat{I}=\frac{1}{2} \hat{I}_{\alpha \beta} \theta^{\alpha} \theta^{\beta}
$$

can be written as

$$
d \hat{I}=0 .
$$

Therefore we see that there must exist a 1 -form $C$ such that

$$
\hat{I}_{\gamma \delta}=e_{[\gamma} C_{\delta]} .
$$

We can now state more precisely the relation between noncommutativity and gravity in the linear approximation. With $c_{\alpha \beta}=0$ we have

$$
\Lambda_{\beta}^{\alpha}=J_{0}^{\alpha \gamma}\left(\hat{I}_{\gamma \beta}+e_{\beta} A_{\gamma}\right) \text {. }
$$


If we denote the perturbation of the metric as

$$
g^{\mu \nu}=\eta^{\mu \nu}-\epsilon g_{1}^{\mu \nu}
$$

we easily derive the relation

$$
g_{1}^{\mu \nu}=-\eta^{\alpha \beta} \Lambda_{\alpha}^{(\mu} \delta_{\beta}^{\nu)}=-\Lambda^{(\mu \nu)} .
$$

It follows from (9) that

$$
g_{1 \alpha \beta}=-J_{0(\alpha}^{\gamma}\left(\hat{I}_{\gamma \beta)}+e_{\beta} A_{\gamma}\right) .
$$

The frame itself is given by

$$
\theta^{\alpha}=d\left(x^{\alpha}-\epsilon J_{0}^{\alpha \gamma} A_{\gamma}\right)-\epsilon J_{0}^{\alpha \gamma} \hat{I}_{\gamma \beta} d x^{\beta} .
$$

We therefore find the following expressions

$$
d \theta^{\alpha}=-\epsilon J_{0}^{\alpha \gamma} e_{\delta} \hat{I}_{\gamma \beta} d x^{\delta} d x^{\beta}=\frac{1}{2} \epsilon J_{0}^{\alpha \delta} e_{\delta} \hat{I}_{\beta \gamma} d x^{\gamma} d x^{\beta}, \quad C^{\alpha \gamma}{ }_{\beta \gamma}=\epsilon J_{0}^{\alpha \delta} e_{\delta} \hat{I}_{\beta \gamma} .
$$

Using the expression

$$
\omega_{\alpha \beta \gamma}=\frac{1}{2}\left(C_{\alpha \beta \gamma}-C_{\beta \gamma \alpha}+C_{\gamma \alpha \beta}\right)
$$

for the components of the connection 1-form $\omega^{\alpha}{ }_{\beta}=\omega^{\alpha}{ }_{\gamma \beta} \theta^{\gamma}$, we find

$$
\omega_{\alpha \beta \gamma}=\frac{1}{2} \epsilon\left(J_{0[\alpha}{ }^{\delta} e_{\delta} \hat{I}_{\beta \gamma]}+J_{0 \beta}{ }^{\delta} e_{\delta} \hat{I}_{\alpha \gamma}\right) .
$$

The torsion obviously vanishes.

Further, using the definition of the Riemann curvature tensor

$$
\Omega_{\beta}^{\alpha}=R_{\beta \gamma \delta}^{\alpha} \theta^{\gamma} \theta^{\delta}=d \omega^{\alpha}{ }_{\beta}+\omega^{\alpha}{ }_{\gamma} \omega_{\beta}^{\gamma}
$$

from (10) we obtain for the linearized curvature

$$
R_{\alpha \beta \gamma \delta}=\frac{1}{2} \epsilon e^{\eta}\left(J_{0 \eta[\gamma} e_{\delta]} \hat{I}_{\alpha \beta}+J_{0 \eta[\alpha} e_{\beta]} \hat{I}_{\gamma \delta}\right) .
$$

For the Ricci curvature we find

$$
R_{\beta \gamma}=-\frac{1}{2} \epsilon e^{\zeta}\left(J_{0 \zeta(\beta} e^{\alpha} \hat{I}_{\gamma) \alpha}+J_{0 \zeta}^{\alpha} e_{(\beta} \hat{I}_{\gamma) \alpha}\right) .
$$

One more contraction yields the expression

$$
R=-2 \epsilon J_{0}^{\zeta \alpha} e_{\zeta} e^{\beta} \hat{I}_{\alpha \beta}
$$

for the Ricci scalar. Using the cocycle condition permits us to write this in the form

$$
R=\epsilon \Delta \chi
$$

where the scalar field $\chi$, the trace component of the perturbation, is defined as

$$
\chi=J_{0}^{\alpha \beta} \hat{I}_{\alpha \beta} .
$$




\section{The WKB approximation}

In the commutative case the WKB dispersion relations follow from the Einstein equations. In order to introduce the WKB approximation in noncommutative case, we suppose that the algebra $\mathcal{A}$ is a tensor product

$$
\mathcal{A}=\mathcal{A}_{0} \otimes \mathcal{A}_{\omega}
$$

of a 'slowly-varying' factor $\mathcal{A}_{0}$ in which all amplitudes lie and a 'rapidly-varying' phase factor which is of order-of-magnitude $\epsilon$ so that only functions linear in this factor can appear. By 'slowly-varying' element $f$ of the algebra we mean an element with a classical limit $\tilde{f}$ such that $\partial_{\alpha} \tilde{f} \lesssim \mu \tilde{f}$. The generic element $f$ of the algebra then is of the form

$$
f=f_{0}+\epsilon \bar{f} e^{i \omega \phi}
$$

where $f_{0}$ and $\bar{f}$ belong to $\mathcal{A}_{0}$. Because of the condition on $\epsilon$ the factor order does not matter and these elements form an algebra. The frequency parameter $\omega$ is so chosen that for an element $f$ of $\mathcal{A}_{0}$ the estimate

$$
[\phi, f] \simeq k \mu
$$

holds. The commutator $\left[f, e^{i \omega \phi}\right]$ is thus of order of magnitude

$$
\left[f, e^{i \omega \phi}\right] \simeq k \mu \omega
$$

The wave vector

$$
\xi_{\alpha}=e_{\alpha} \phi
$$

is normal to the surfaces of constant phase. We shall require also that the energy of the wave be such that it contribute not as source to the background field. This inequality can be written as

$$
\epsilon \omega^{2} \ll \mu^{2} .
$$

It also assures us that for the approximation we are considering we need not pay attention to the order of the factors in the perturbation. We have in fact partially solved the system of equations without further approximation. The purpose of the following analysis is to find the constraints on the wave vector $\xi$.

\subsection{The quasi-commutative case}

In the WKB approximation the perturbations $\Lambda_{\beta}^{\alpha}$ and $I^{\alpha \beta}$ are of the form

$$
\Lambda_{\beta}^{\alpha}=\bar{\Lambda}_{\beta}^{\alpha} e^{i \omega \phi}, \quad I^{\alpha \beta}=\bar{I}^{\alpha \beta} e^{i \omega \phi},
$$

where $\bar{\Lambda}_{\beta}^{\alpha}$ and $\bar{I}^{\alpha \beta}$ belong to $\mathcal{A}_{0}$. Therefore we have also

$$
g_{1}^{\mu \nu}=\bar{g}^{\mu \nu} e^{i \omega \phi} .
$$

Using $\xi_{\alpha}$ and $\eta^{\alpha}=J_{0}^{\alpha \beta} \xi_{\beta}$ we have

$$
e_{\alpha} I_{\beta \gamma}=\left(i \omega \xi_{\alpha} \bar{I}_{\beta \gamma}+e_{\alpha} \bar{I}_{\beta \gamma}\right) e^{i \omega \phi}, \quad e_{\alpha} \Lambda_{\beta \gamma}=\left(i \omega \xi_{\alpha} \bar{\Lambda}_{\beta \gamma}+e_{\alpha} \bar{\Lambda}_{\beta \gamma}\right) e^{i \omega \phi} .
$$


The cocycle condition replaces Einstein equation to a certain extent. In the WKB approximation it becomes

$$
\xi_{\alpha} \hat{I}_{\beta \gamma}+\xi_{\beta} \hat{I}_{\gamma \alpha}+\xi_{\gamma} \hat{I}_{\alpha \beta}=0
$$

We multiply this equation by $\xi^{\alpha}$ and obtain

$$
\xi^{2} \hat{I}_{\beta \gamma}+\xi_{[\beta} \hat{I}_{\gamma] \alpha} \xi^{\alpha}=0
$$

If $\xi^{2} \neq 0$ then we conclude that

$$
\hat{I}_{\beta \gamma}=-\xi^{-2} \xi_{[\beta} \hat{I}_{\gamma] \alpha} \xi^{\alpha} .
$$

This is no restriction; it defines simply $C_{\alpha}$ by

$$
i \omega C_{\alpha}=-\xi^{-2} \hat{I}_{\alpha \beta} \xi^{\beta}
$$

If $\xi^{2}=0$ then we conclude that

$$
\xi_{[\beta} \hat{I}_{\gamma] \alpha} \xi^{\alpha}=0
$$

This is a small restriction; the $\xi_{\alpha}$ must be a Petrov vector of $\hat{I}$. We shall improve this in a particular case in the next section. In terms of the scalar $\chi$ we obtain the relation

$$
\hat{I}_{\alpha \beta} \eta^{\beta}=-\frac{1}{2} \chi \xi_{\alpha}
$$

Using the definition of $\eta$ we find in the WKB approximation to first order

$$
\begin{aligned}
& \omega_{\alpha \beta \gamma}=\frac{1}{2} \epsilon(i \omega)\left(\eta_{[\alpha} \hat{I}_{\beta \gamma]}+\eta_{\beta} \hat{I}_{\alpha \gamma}\right), \\
& R_{\alpha \beta \gamma \delta}=-\frac{1}{2} \epsilon(i \omega)^{2}\left(\eta_{[\gamma} \xi_{\delta]} \hat{I}_{\alpha \beta}-\eta_{[\alpha} \xi_{\beta]} \hat{I}_{\gamma \delta}\right), \\
& R_{\beta \gamma}=-\frac{1}{2} \epsilon(i \omega)^{2}\left(\xi_{(\beta} \eta^{\alpha}-\xi^{\alpha} \eta_{(\beta}\right) \hat{I}_{\gamma) \alpha}, \\
& R=\epsilon(i \omega)^{2} \chi \xi^{2} .
\end{aligned}
$$

In average the terms linear in $\epsilon$ vanish. Therefore in principle we have to calculate to second order and average over several wavelengths. Using

$$
\left\langle\hat{I}^{\alpha \beta}\right\rangle=0, \quad\left\langle\hat{I}^{\alpha \beta} \hat{I}^{\gamma \delta}\right\rangle=\frac{1}{2} \hat{\bar{I}}^{\alpha \beta} \hat{\bar{I}}^{\gamma \delta}
$$

and expanding the curvature to second order we find the expression

$$
\left\langle R_{\beta \gamma}\right\rangle=\frac{1}{2} \epsilon^{2}(i \omega)^{2}\left(\bar{\chi} \xi^{\alpha} \eta_{(\gamma} \hat{\bar{I}}_{\beta) \alpha}+\frac{3}{4} \bar{\chi}^{2} \xi_{\beta} \xi_{\gamma}+\eta^{2} \hat{\bar{I}}_{\eta \beta} \hat{\bar{I}}_{\gamma}^{\eta}-\frac{1}{2} \eta_{\beta} \eta_{\gamma} \hat{\bar{I}}_{\alpha \eta} \hat{\bar{I}}^{\alpha \eta}\right)
$$

for the Ricci tensor and the expression

$$
\langle R\rangle=\frac{1}{8} \epsilon^{2}(i \omega)^{2}\left(2 \eta^{2} \hat{\bar{I}}_{\alpha \beta} \hat{\bar{I}}^{\alpha \beta}+7 \bar{\chi}^{2} \xi^{2}\right)
$$

for the Ricci scalar. We shall return to these formulae in Section 5. 


\subsection{The noncommutative lattice}

As a lattice, the background noncommutativity is of considerable complexity, the contrary of a simple cubic lattice. It is in general non-periodic but in the WKB approximation we can assume periodicity since at the scale of the frequency $J_{0}^{\mu \nu}$ is a constant $4 \times 4$ matrix. It is difficult to obtain general expressions for the modes of the high frequency waves and their dispersion relations; however, it is interesting to analyse them in more detail by considering a specific example. We take an arbitrary perturbation $\hat{I}_{\alpha \beta}$ with the wave vector $\xi_{\alpha}$ normalized so that $\xi_{0}=-1$,

$$
\hat{\bar{I}}_{\alpha \beta}=\left(\begin{array}{cccc}
0 & b_{3} & -b_{2} & e_{1} \\
-b_{3} & 0 & b_{1} & e_{2} \\
b_{2} & -b_{1} & 0 & e_{3} \\
-e_{1} & -e_{2} & -e_{3} & 0
\end{array}\right) .
$$

One easily sees that the cocycle condition is equivalent to the constraint $\vec{b}=-\vec{\xi} \times \vec{e}$ which is the part of the field equations for the electromagnetic plane wave, the Bianchi equations. Suppose that $\xi$ is null and oriented along the $z$-axis, $\xi_{\alpha}=(0,0,1,-1)$. The cocycle condition imposes that $\hat{I}$ equals to

$$
\hat{\bar{I}}_{\alpha \beta}=\left(\begin{array}{cccc}
0 & 0 & -e_{1} & e_{1} \\
0 & 0 & -e_{2} & e_{2} \\
e_{1} & e_{2} & 0 & e_{3} \\
-e_{1} & -e_{2} & -e_{3} & 0
\end{array}\right) .
$$

The perturbation $\hat{I}$ is of Petrov-type $N$ if $\vec{\xi} \cdot \vec{e}=e_{3}=0$; this would be the second half of the Maxwell field equations. In this case, for an arbitrary background noncommutativity given by

$$
J_{0 \alpha \beta}=\left(\begin{array}{cccc}
0 & B_{3} & -B_{2} & E_{1} \\
-B_{3} & 0 & B_{1} & E_{2} \\
B_{2} & -B_{1} & 0 & E_{3} \\
-E_{1} & -E_{2} & -E_{3} & 0
\end{array}\right)
$$

we can write the amplitude of the metric perturbation in the form

$$
\bar{g}_{1 \alpha \beta}=-J_{0(\alpha}{ }^{\gamma} \hat{\bar{I}}_{\gamma \beta)}=\left(\begin{array}{cc}
P_{11} & P_{12} \\
P_{12}^{T} & P_{22}
\end{array}\right) .
$$

The expressions for $P_{12}, P_{22}$ can be obtained from (17)-(18) but they are somewhat lengthy. However, it is easy to check that by a change of coordinates we can set $P_{12}=0, P_{22}=0$. Introducing $e_{1}=a \cos \gamma, e_{2}=a \sin \gamma, B_{2}+E_{1}=A \sin \Gamma, B_{1}-E_{2}=A \cos \Gamma$ the remaining part $P_{11}$ can be decomposed

$$
P_{11}=a A\left(\begin{array}{cc}
\sin (\gamma+\Gamma) & \cos (\gamma+\Gamma) \\
\cos (\gamma+\Gamma) & -\sin (\gamma+\Gamma)
\end{array}\right)+a A\left(\begin{array}{cc}
\sin (\gamma-\Gamma) & 0 \\
0 & \sin (\gamma-\Gamma)
\end{array}\right)
$$

into a trace-free part and a trace. The trace-free part corresponds to a gravitational wave which is polarized, and though the polarization is fixed in terms of $\gamma+\Gamma$, it can be arbitrary. In

addition there is a scalar wave, the trace. In the case when $e_{3} \neq 0$ the perturbation $\hat{I}$ is not of Petrov type $N$; the additional gravitational mode is longitudinal, a constraint mode. 


\section{The Poisson energy and conservation laws}

We have in fact associated a gravitational field to the noncommutative structure with the map (7). We would like to consider now this structure as an effective field and estimate its energy-momentum which we call the 'Poisson energy'. We are confronted immediately with the choice of the position of the extra term in the Einstein equations. If we place it on the right-hand side, we can consider it as an effective matter source. If we keep it on the left-hand side then we interpret it as a noncommutative modification of the curvature. First however we make some preliminary remarks about conservation laws.

From (11)-(12) for the Einstein tensor we obtain

$$
G_{\beta \gamma}=-\frac{1}{2} \epsilon\left(J_{0 \zeta(\beta} e^{\zeta} e^{\alpha} \hat{I}_{\gamma) \alpha}+J_{0}^{\alpha \zeta} e_{\zeta} e_{(\beta} \hat{I}_{\gamma) \alpha}-2 \eta_{\beta \gamma} J_{0 \zeta \delta} e^{\zeta} e_{\alpha} \hat{I}^{\delta \alpha}\right) .
$$

In general the Einstein tensor does not vanish. A conservation equation of the associated energymomentum tensor in linear approximation is easy to verify. Applying the cocycle condition and keeping in mind that, to linear order in $\epsilon, e_{\alpha} e_{\beta}=e_{\beta} e_{\alpha}$, we obtain

$$
\begin{aligned}
e^{\beta} G_{\beta \gamma} & =-\frac{1}{2} \epsilon\left(J_{0}^{\alpha \zeta} e^{\beta} e_{\zeta} e_{\gamma} \hat{I}_{\beta \alpha}+J_{0}^{\alpha \zeta} e^{\beta} e_{\zeta} e_{\beta} \hat{I}_{\gamma \alpha}-2 J_{0 \zeta \delta} e_{\gamma} e^{\zeta} e_{\alpha} \hat{I}^{\delta \alpha}\right) \\
& =-\frac{1}{2} \epsilon J_{0} \delta \zeta e_{\zeta} e^{\alpha}\left(e_{\alpha} \hat{I}_{\gamma \delta}-e_{\gamma} \hat{I}_{\alpha \delta}\right)=\frac{1}{2} \epsilon J_{0 \delta \zeta} e^{\zeta} e^{\alpha} e^{\delta} \hat{I}_{\alpha \gamma}=0 .
\end{aligned}
$$

As we shall see, the conservation law holds in an important special case in quadratic order too.

\subsection{Canonical orientation}

To the extent that the noncommutative background is analogous to a lattice, the perturbations can be considered as elastic vibrations or phonons. This analogy however is tenuous at the approximation we are considering since we have excluded any resonance phenomena. These could appear if we allowed larger-amplitude waves with energy sufficient to change the background. The case we shall now focus to would then be analogous to a phonon propagating along one of the axes of a regular cubic lattice. In the special case in which it is also Petrov vector of the perturbation the dispersion relations become clearer.

Assume then that $\eta$ and $\xi$ are parallel and set

$$
\eta^{\alpha}=J_{0}^{\alpha \beta} \xi_{\beta}=\lambda \xi^{\alpha}
$$

It follows from (14) that the vector $\xi$ is an eigenvector of $J$ not only to first but also to second order. Equation (15) yields for the linearized Riemann curvature

$$
R_{\alpha \beta \gamma \delta}=0
$$

The dispersion relation

$$
\xi^{2}=0
$$

follows from (13).

In quadratic order, using the dispersion relation, we find that the expression (16) for the Ricci tensor simplifies to

$$
\left\langle R_{\beta \gamma}\right\rangle=-\frac{1}{8} \epsilon^{2}(i \omega)^{2}\left(\bar{\chi}^{2}+2 \lambda^{2} \hat{\bar{I}}_{\alpha \eta} \hat{\bar{I}}^{\alpha \eta}\right) \xi_{\beta} \xi_{\gamma} .
$$

The Ricci scalar vanishes and we obtain for the Einstein tensor the average value

$$
\left\langle G_{\beta \gamma}\right\rangle=-\rho \xi_{\beta} \xi_{\gamma}
$$


with

$$
\rho=-\frac{1}{8}(\epsilon \omega)^{2}\left(\bar{\chi}^{2}+2 \lambda^{2} \hat{\bar{I}}_{\alpha \eta} \hat{\bar{I}}^{\alpha \eta}\right) .
$$

The energy-momentum is that of a null dust with a density $\rho$.

In the WKB approximation we can, just as in the classical case, derive a conservation law for $\rho$ which has a natural interpretation as graviton-number conservation. If we multiply the cocycle condition (8) by $\xi^{\alpha}$ we obtain

$$
\xi^{\alpha} e_{\alpha} \hat{I}_{\beta \gamma}+\xi^{\alpha} e_{\beta} \hat{I}_{\gamma \alpha}+\xi^{\alpha} e_{\gamma} \hat{I}_{\alpha \beta}=0 .
$$

We also have

$$
e^{\alpha}\left(\xi_{\alpha} \hat{I}_{\beta \gamma}+\xi_{\beta} \hat{I}_{\gamma \alpha}+\xi_{\gamma} \hat{I}_{\alpha \beta}\right)=0 .
$$

Adding these two equations, using (14), (19) and not forgetting that $e_{\alpha} \xi_{\beta}=e_{\beta} \xi_{\alpha}$ in our approximation, we find

$$
\begin{gathered}
\left(\xi^{\alpha} e_{\alpha} \hat{I}_{\beta \gamma}+e^{\alpha}\left(\xi_{\alpha} \hat{I}_{\beta \gamma}\right)\right) \hat{I}^{\beta \gamma}+2\left(\xi^{\alpha} e_{\beta} \hat{I}_{\gamma \alpha}+e^{\alpha}\left(\xi_{\beta} \hat{I}_{\gamma \alpha}\right)\right) \hat{I}^{\beta \gamma} \\
=e_{\alpha}\left(\xi^{\alpha} \hat{I}^{\beta \gamma} \hat{I}_{\beta \gamma}\right)+2 e^{\alpha}\left(\xi^{\beta} \hat{I}^{\gamma \alpha} \hat{I}_{\beta \gamma}\right)=0 .
\end{gathered}
$$

The conservation law

$$
e^{\alpha}\left(\rho \xi_{\alpha}\right)=0
$$

follows and from it the conservation of the effective source,

$$
e^{\alpha}\left(\rho \xi_{\alpha} \xi_{\beta}\right)=0 .
$$

To interpret the additional term we have isolated as the energy-momentum of an external field,

$$
G_{\beta \gamma}=-16 \pi G_{N} T_{\beta \gamma}
$$

the sign of $\rho$ should be non-negative. However, as

$$
\rho=-\frac{1}{8}(\epsilon \omega)^{2}\left(\bar{\chi}^{2}+2 \lambda^{2} \hat{\bar{I}}_{\alpha \eta} \hat{\bar{I}}^{\alpha \eta}\right)=\frac{1}{4}(\epsilon \omega \lambda \vec{e})^{2}-\frac{1}{4}(\epsilon \omega \lambda \vec{b})^{2}-\frac{1}{8}(\epsilon \omega \bar{\chi})^{2},
$$

the matter density does not have a fixed sign, unless of course one place restrictions on the relative importance of the space-time and space-space commutation relations. This exactly is one of the properties which could explain the acceleration of the universe [4] and it makes the 'Poisson energy' a possible candidate for dark energy. We shall examine this in more detail in the future work.

\section{Conclusions}

The formalism on which the article has been based is one with a preferred frame. It is in a sense gauge-fixed from the beginning. We have shown that the degrees-of-freedom or basic modes of the resulting theory of gravity can be put in correspondence with those of the noncommutative structure. As an application of the formalism we have considered a high-frequency perturbation of the metric. In the classical theory it follows from the field equations that the perturbation must satisfy a dispersion relation and a conservation law. We show that these remain valid in the 
noncommutative extension of the frame formalism and that they are consequences of a cocycle condition on the corresponding perturbation of the Poisson structure.

The analysis of the dispersion relations however shows that the content of the high-frequency radiation is not identical in the two cases. Noncommutative gravity accommodates the Einsteinean gravitational waves but they get necessarily polarized by the background noncommutative lattice. There is also a massless scalar mode. In addition, we obtain that massive longitudinal modes can exist; it is reasonable however to expect that they would be eliminated by some additional equations of motion for the Poisson structure.

We have also shown that the perturbation of the Poisson structure contributes to the energymomentum as an additional effective source of the gravitational field. Although the explicit form of this contribution, the Poisson energy, was calculated only in a linearized, high-frequency approximation it is certainly significant in a more general context. It would be very interesting to examine the properties the Poisson energy beyond the WKB approximation, in particular in the context of cosmology.

It would be nice, as a referee of this paper has suggested, to compare the details of the frame approach to noncommutative gravity to the other approaches, e.g. $[5,6,7,8]$; we will here point out the main differences. A careful reader has already noticed that the noncommutative frame formalism has intrinsically geometric formulation. The metric, connection, curvature are all defined via the forms; they have the usual differential-geometric properties as e.g. linearity, derivatives obey the Leibniz rule etc. As a consequence, inbuilt in the theory are the usual symmetries as coordinate invariance; the commutator $J^{\mu \nu}$ can have arbitrary dependence on the coordinates. On the other hand, the analysis as presented is representation-free and thus we have no action or equations of motion: all relations are just algebraic constraints.

The approach developed in $[5,6,7,8]$ emphasizes the gauge-field properties of the vielbein and connection and follows the logic of the field theory. Thus basically one needs the representation of the noncommutative fields (usually, the Moyal-Weyl representation with the commutator $J^{\mu \nu}=$ const). Some properties of the differential calculus have to be changed, for example the Leibniz rule. Symmetries however are well defined and the action principle can be postulated. The two approaches, clearly, differ conceptually and maybe the best way to compare them is to compare the corresponding solutions to the specific physical problems. In this spirit, we present our results for the gravitational wave propagation here.

\section{Acknowledgements}

This work is supported by the EPEAEK programme "Pythagoras II" and co-funded by the European Union(75\%) and the Hellenic state (25\%). A CEI grant for participation in the Seventh International Conference "Symmetry in Nonlinear Mathematical Physics" is gratefully acknowledged.

\section{References}

[1] Madore J., An introduction to noncommutative differential geometry and its physical applications, 2nd ed., London Mathematical Society Lecture Note Series, no. 257, Cambridge University Press, 2000.

[2] Burić M., Madore J., A dynamical 2-dimensional fuzzy space, Phys. Lett. B 622 (2005), 183-191, hep-th/0507064.

[3] Burić M., Grammatikopoulos T., Madore J., Zoupanos G., Gravity and the structure of noncommutative algebras, J. High Energy Phys. 2006 (2006), no. 04, 054, 17 pages, hep-th/0603044.

[4] Sahni V., Starobinsky A., Reconstructing dark energy, Internat. J. Modern Phys. D 15 (2006), 2105-2132, astro-ph/0610026.

[5] Cardella M.A., Zanon D., Noncommutative deformation of four dimensional Einstein gravity, Classical Quantum Gravity 20 (2003), L95-L104, hep-th/0212071. 
[6] Garcia-Compean H., Obregon O., Ramirez C., Sabido M., Noncommutative self-dual gravity, Phys. Rev. D 68 (2003), 044015, 8 pages, hep-th/0302180.

[7] Aschieri P., Blohmann C., Dimitrijevic M., Meyer F., Schupp P., Wess J., A gravity theory on noncommutative spaces, Classical Quantum Gravity 22 (2005), 3511-3532, hep-th/0504183.

[8] Aschieri P., Dimitrijevic M., Meyer F., Wess J., Noncommutative geometry and gravity, Classical Quantum Gravity 23 (2006), 1883-1912, hep-th/0510059. 